\title{
Altered function of nitrergic nerves inhibiting sympathetic neurotransmission in mesenteric vascular beds of renovascular hypertensive rats
}

\author{
Toshihiro Koyama ${ }^{1}$, Yukako Hatanaka ${ }^{1}$, Xin Jin $^{1}$, Ayako Yokomizo ${ }^{1}$, Hidetoshi Fujiwara ${ }^{1}$, Mitsuhiro Goda ${ }^{1}$, \\ Narumi Hobara ${ }^{2}$, Yoshito Zamami ${ }^{1}$, Yoshihisa Kitamura ${ }^{3}$ and Hiromu Kawasaki ${ }^{1}$
}

Neuronal nitric oxide (NO) has been shown to modulate perivascular adrenergic neurotransmission by inhibiting noradrenaline release from terminals in rat mesenteric arteries. This study was conducted to investigate changes in the inhibitory function of NO-containing nerves (nitrergic nerves) in mesenteric vascular beds of 2-kidney, 1-clip renovascular hypertensive rats (2K1C-RHR). Rat mesenteric vascular beds without endothelium were perfused with Krebs solution and the perfusion pressure was measured. In preparations from sham-operated rats (control) and 2K1C-RHRs, vasoconstriction induced by periarterial nerve stimulation (PNS; $2-8 \mathrm{~Hz}$ ), but not vasoconstriction induced by exogenously injected noradrenaline $(0.5,1.0 \mathrm{nmol})$, was markedly facilitated in the presence of a nonselective NO synthase (NOS) inhibitor, $\mathrm{N}$ - $\omega$-nitro-L-arginine methyl ester (L-NAME) $(100 \mu \mathrm{M})$. The facilitatory effect of L-NAME in preparations from 2K1C-RHR was smaller than that in control preparations. L-NAME augmented PNS-evoked noradrenaline release, which was smaller in 2K1C-RHRs than in controls. The expression of neuronal NO synthase (nNOS) measured by western blotting in mesenteric arteries from 2K1C-RHRs was significantly decreased compared with control arteries. Immunohistochemical staining of mesenteric arteries showed dense innervation of nNOSimmunopositive nerves that was significantly smaller in arteries from 2K1C-RHR than that in control arteries. Mesenteric arteries were densely innervated by tyrosine hydroxylase-immunopositive nerves, which coalesced with nNOS-immunopositive nerves. These results suggest that the inhibitory function of nitrergic nerves in adrenergic neurotransmission is significantly decreased in 2K1C-RHRs. This functional alteration based on the decrease in nNOS expression and nitrergic innervation leads to enhanced adrenergic neurotransmission and contributes to the initiation and development of renovascular hypertension. Hypertension Research (2010) 33, 485-491; doi:10.1038/hr.2010.48; published online 9 April 2010

Keywords: adrenergic neurotransmission; neuronal nitric oxide; nitrergic nerve innervation; rat mesenteric artery; renovascular hypertension

\section{INTRODUCTION}

Although it is widely accepted that peripheral vascular tone is mainly maintained by the perivascular adrenergic nervous system, accumulating findings show that nonadrenergic noncholinergic nerves innervating blood vessels have a role in vascular tone control. ${ }^{1,2}$ Nitric oxide (NO), a potent vasodilator, is synthesized from L-arginine by $\mathrm{NO}$ synthase (NOS) in various cells. NOS is present not only in vascular endothelial cells (endothelial NOS), but also in perivascular nerves (neuronal NOS; nNOS), ${ }^{3,4}$ and the NO synthesized by nNOS has been shown to act as a neurotransmitter to produce strong vasodilation. ${ }^{2}$ Thus, perivascular NO-containing nerves (nitrergic nerves) have been shown to distribute in various blood vessels as nonadrenergic noncholinergic nerves. ${ }^{5-7}$
In addition to NO's direct vasodilator effect, it has also been shown to modulate adrenergic neurotransmission. ${ }^{8,9}$ Our previous study showed that NO, which is released from nitrergic nerves, presynaptically inhibits neurogenic noradrenaline release to modulate adrenergic neurotransmission in rat mesenteric arteries without an endothelium. ${ }^{6}$ Furthermore, several studies have shown that inhibition of NO synthesis results in augmented adrenergic nerve-mediated vasoconstriction in the rat tail artery, ${ }^{10}$ the large coronary artery of anesthetized $\operatorname{dogs}^{11}$ and the isolated adrenal medulla vessels of dogs. ${ }^{8}$ These findings imply that NO has a role in the modulation of adrenergic neurotransmission.

Increased activity of the adrenergic nervous system has been implicated in the initiation and development of hypertension, as

\footnotetext{
1Department of Clinical and Pharmaceutical Science, Graduate School of Medicine, Dentistry and Pharmaceutical Sciences, Okayama University, Tsushima-naka, Okayama, Japan; ${ }^{2}$ Department of Life Science, Okayama University of Science, Ridai-cho, Okayama, Japan and ${ }^{3}$ Pharmaceutical Care and Health, Graduate School of Medicine, Dentistry and Pharmaceutical Sciences, Okayama University, Tsushima-naka, Okayama, Japan

Correspondence: Professor H Kawasaki, Department of Clinical Pharmaceutical Science, Graduate School of Medicine, Dentistry and Pharmaceutical Sciences, Okayama University, 1-1-1 Tsushima-naka, North Ward, Okayama 700-8530, Japan.

E-mail: kawasaki@pheasant.pharm.okayama-u.ac.jp

Received 30 October 2009; revised 25 December 2009; accepted 22 January 2010; published online 9 April 2010
} 
enhanced adrenergic neurotransmission has been observed in hypertensive patients ${ }^{12,13}$ as well as in various experimental models such as the spontaneously hypertensive rat (SHR), ${ }^{14}$ the 2-kidney, 1-clip renovascular hypertensive rats $(2 \mathrm{~K} 1 \mathrm{C}-\mathrm{RHR})^{15}$ and other experimental hypertensive animals. ${ }^{16,17}$ However, the precise causal mechanisms leading to adrenergic nervous system enhancement are controversial. Thus, it is assumed that the function of nitrergic nerves, which inhibit adrenergic neurotransmission, may be altered in hypertension.

Therefore, the aim of this study was to observe changes in NOmediated modulation of adrenergic neurotransmission in the mesenteric vascular bed of an experimental hypertensive model, 2K1C-RHR. To examine functional alteration, we examined changes in adrenergic nerve-mediated vascular responses to periarterial nerve stimulation (PNS) in the presence of a nonselective NOS inhibitor, $N$ - $\omega$-nitro-Larginine methyl ester (L-NAME), which inhibits nitrergic nerve function, in mesenteric vascular beds without an endothelium. Furthermore, we investigated the expression of nNOS and the distribution of nitrergic nerves in the mesenteric artery. This study showed that the inhibitory function of nitrergic nerves on adrenergic neurotransmission is significantly decreased in $2 \mathrm{~K} 1 \mathrm{C}-\mathrm{RHRs}$, leading to enhanced adrenergic neurotransmission and contributing to the initiation and development of renovascular hypertension in 2K1C-RHRs.

\section{METHODS}

\section{Animals}

Six-week-old male Wistar rats (purchased from Shimizu Experimental Animal, Shizuoka, Japan) were used in this study. The animals were given food and water ad libitum. The animals were housed in the Animal Research Center of Okayama University at a controlled ambient temperature of $25^{\circ} \mathrm{C}$ with $50 \pm 10 \%$ relative humidity and a 12 -h day night ${ }^{-1}$ cycle (lights on at 8:00 AM). This study was carried out in accordance with the Guidelines for Animal Experiments at Okayama University Advanced Science Research Center, the Japanese Government Animal Protection and Management Law (No. 115) and the Japanese Government Notification on Feeding and Safekeeping of Animals (No. 6). Every effort was made to minimize the number of animals used and their suffering.

\section{Surgical procedure}

With the rats under anesthesia with sodium pentobarbital $\left(50 \mathrm{mg} \mathrm{kg}^{-1}\right.$, intraperitoneally) (Sigma Aldrich Japan, Tokyo, Japan), the right renal artery was carefully isolated and clipped with a silver-ribbon clip $(2.0 \mathrm{~mm}$ wide with a $0.2 \mathrm{~mm}$ slit width); the left renal artery was left intact. To examine the influence of the operation, sham-operated rats underwent the same surgical procedures without clipping the renal artery. After clipping, an antibiotic (penicillin G; Sigma Aldrich Japan) was infused around the surgical area and the incision was closed. After the operation, the animals were transferred into individual cages in a temperature-controlled room.

\section{Systolic blood pressure and heart rate measurements}

Systolic blood pressure and heart rate of conscious rats were measured before the operation and 4 weeks after the operation by tail-cuff plethysmography (model TK-370C; UNICOM, Tokyo, Japan). Animals with systolic blood pressures of more than $150 \mathrm{~mm} \mathrm{Hg}$ were classed as 2K1C-RHRs and were used in the following experiment.

\section{Perfusion of mesenteric vascular beds and perfusion pressure measurement}

The sham-operated rats and 2K1C-RHRs at 10-weeks old were anesthetized with sodium pentobarbital ( $50 \mathrm{mg} \mathrm{kg}^{-1}$, intraperitoneally), and the mesenteric vascular beds were isolated and prepared for perfusion as described earlier. ${ }^{18,1}$ Briefly, the superior mesenteric artery was cannulated with a polyethylene tube, and the entire intestine-associated vascular bed was removed. The mesenteric vascular bed was separated from the intestine by cutting close to the intestinal wall and prepared for perfusion. The isolated mesenteric vascular bed was perfused with a modified (see below) Krebs-Ringer bicarbonate solution (Krebs solution) at a constant flow rate of $5 \mathrm{ml} \mathrm{min}^{-1}$ with a peristaltic pump (model AC-2120, ATTO, Tokyo, Japan) and superfused with the same solution at a rate of $0.5 \mathrm{ml} \mathrm{min}^{-1}$ to prevent drying. The Krebs solution was bubbled with a mixture of $95 \% \mathrm{O}_{2}$ and $5 \% \mathrm{CO}_{2}$. The modified Krebs solution had the following composition (mM): $\mathrm{NaCl} 119.0, \mathrm{KCl} 4.7, \mathrm{CaCl}_{2}$ 2.4, $\mathrm{MgSO}_{4}$ 1.2, $\mathrm{NaHCO}_{3}$ 25.0, $\mathrm{KH}_{2} \mathrm{PO}_{4}$ 1.2, EDTA-2Na 0.03 and glucose $11.1(\mathrm{pH} 7.4)$. Changes in the perfusion pressure were measured with a pressure transducer (model TP-400T, Nihon Kohden, Tokyo, Japan) and recorded using a pen recorder (model U-228, Nippon Denshi Kagaku, Tokyo, Japan).

\section{Chemical removal of the vascular endothelium}

To remove the vascular endothelium, preparations with resting tone were perfused with $1.80 \mathrm{mg} \mathrm{ml}^{-1}$ solution of sodium deoxycholate (Sigma Aldrich Japan) in saline for $30 \mathrm{~s}$ as described earlier. ${ }^{4,19}$ The preparations were then rinsed with a sodium deoxycholate-free Krebs solution containing a selective $\alpha_{1}$-adrenoceptor agonist, methoxamine (1-2 $\mu \mathrm{m}$, Nippon Shinyaku, Kyoto, Japan), and chemical removal of the endothelium was assessed by the lack of a relaxant effect in active tone after a bolus injection of $1 \mathrm{nmol}$ acetylcholine, which was directly injected into the perfusate proximal to the arterial cannula with an infusion pump (model 975, Harvard Apparatus, Holliston, MA, USA). A volume of $100 \mu \mathrm{l}$ was injected over a period of $12 \mathrm{~s}$.

\section{PNS and bolus injection of noradrenaline}

After allowing the basal perfusion pressure to stabilize, the preparation was initially subjected to PNS at 2, 4 and $8 \mathrm{~Hz}$ and bolus injections of noradrenaline $(0.5,1.0 \mathrm{nmol}$, Daiichi-Sankyo, Tokyo, Japan). PNS was applied using bipolar platinum ring electrodes placed around the superior mesenteric artery. Rectangular pulses of $1 \mathrm{~ms}$ and supramaximal voltage $(50 \mathrm{~V})$ were given for $30 \mathrm{~s}$ using an electronic stimulator (model SEN 3301, Nihon Kohden). Noradrenaline was directly injected into the perfusate proximal to the arterial cannula with an infusion pump (model 975, Harvard Apparatus). A volume of $100 \mu \mathrm{l}$ was injected over a period of $12 \mathrm{~s}$.

\section{Experimental protocols for vascular responses}

After responses to the first PNS $\left(\mathrm{S}_{1}\right)$ and noradrenaline injection $\left(\mathrm{I}_{1}\right)$ were obtained as controls, the Krebs solution was switched to Krebs solution containing L-NAME ( $100 \mu \mathrm{M}$, Sigma Aldrich Japan); after $20 \mathrm{~min}$, the perfusion of the second PNS $\left(\mathrm{S}_{2}\right)$ and noradrenaline injection $\left(\mathrm{I}_{2}\right)$ were given in the presence of L-NAME. To estimate the effect of the agent tested, changes in perfusion pressure in response to PNS or noradrenaline injection were expressed as the ratio between the vasoconstriction induced by $S_{2}$ and $S_{1}$ or $I_{2}$ and $I_{1}$, respectively.

At the end of each experiment, the preparations were perfused with Krebs solution containing 1-2 $\mu \mathrm{M}$ methoxamine to produce vascular tone, and successful removal of the endothelium was assessed by verifying the lack of a relaxant effect after a bolus injection of $1 \mathrm{nmol}$ acetylcholine.

\section{Measurement of noradrenaline in the perfusate}

In preparations with resting tone and without an endothelium, the perfusate was collected before and after $8 \mathrm{~Hz}$ PNS $\left(S_{1}\right.$ and $\left.S_{2}\right)$ for $3 \mathrm{~min}$. Noradrenaline in the perfusate was absorbed into alumina, and the extract obtained with acetic acid was assayed by high-performance liquid chromatography with electrochemical detection (model HTEC-500, EICOM, Kyoto, Japan); 3, 4-dihydroxybenzylamine hydrobromide (Sigma Aldrich Japan) was used as the internal standard.

\section{Western blot analysis}

The mesenteric arteries isolated from 2K1C-RHR and sham-operated rats at 4 weeks after the operation were homogenized and centrifuged $(10000 \mathrm{~g}, 20 \mathrm{~min}$, $4{ }^{\circ} \mathrm{C}$ ). The concentration of protein in the homogenate was determined using the Bio-Rad protein assay solution (Bio-Rad Laboratories, Osaka, Japan). For western blotting, membrane proteins were electrophoresed and transferred onto a Hybond-P membrane (GE Healthcare UK, Buckinghamshire, UK). 
The membrane was blocked in a blocking buffer (phosphate-buffered saline (PBS) containing $10 \%$ goat serum and $0.01 \mathrm{M}$ EDTA) and then probed with a polyclonal antibody against nNOS (Zymed Laboratories, Carlsbad, CA, USA) (1:1000) or a polyclonal antibody against $\beta$-actin (Cell Signaling Technology, Danvers, MA, USA) (1:2000) in blocking buffer. The washed membrane was incubated with goat anti-rabbit IgG conjugated with horseradish peroxidase (1:1000; R\&D Systems, Minneapolis, MN, USA). Bound antibodies were detected using a chemiluminescent substrate kit (GE Healthcare UK), and the content of $\beta$-actin was used as a control to ensure that the same amount of protein was loaded in each lane.

\section{Immunohistochemical study}

The mesenteric artery was isolated, fixed and immersed as described earlier. ${ }^{20,21}$ Briefly, the third branch of the mesenteric artery was immersion fixed in the Zamboni solution for $48 \mathrm{~h}$. After fixation, the artery was immersed in PBS containing $0.5 \%$ Triton X-100 overnight and incubated with PBS containing normal goat serum (1:100) for $60 \mathrm{~min}$. The tissue was then incubated with rabbit polyclonal anti-nNOS (Zymed Laboratories) antiserum at a dilution of 1:500 for $72 \mathrm{~h}$ at $4{ }^{\circ} \mathrm{C}$. The sites of the antigen-antibody reaction were detected by incubation with fluorescein-5-isothiocyanate-labeled goat anti-rabbit IgG (diluted 1:100) (ICN Pharmaceuticals, Aurora, OH, USA) for $60 \mathrm{~min}$. Immunofluorescence in the arteries was observed under a confocal laser-scanning microscope (CLSM510, Carl Zeiss GmbH, Jena, Germany) in the Okayama University Medical School Central Research Laboratory.

\section{Double immunostaining}

The mesenteric artery was isolated, fixed and immersed as described earlier. ${ }^{20,21}$ The third branch of the mesenteric artery was immersion fixed in the Zamboni solution, immersed in PBS containing 0.5\% Triton X-100 and incubated with PBS containing normal goat serum (1:100). The tissue was then incubated with a solution of mixed rabbit polyclonal anti-nNOS (Zymed Laboratories) antiserum at a dilution of 1:500 with mouse monoclonal anti-tyrosine hydroxylase (TH) (Chemicon, Temecula, CA, USA) antiserum at a dilution of 1:100 for $72 \mathrm{~h}$ at $4{ }^{\circ} \mathrm{C}$. The sites of the antigen-antibody reaction were detected by incubation with a solution of mixed fluorescein-5-isothiocyanate-labeled goat anti-rabbit IgG (diluted 1:100) (ICN Pharmaceuticals) with Cy3-labeled goat anti-mouse IgG (diluted 1:200) (GE Healthcare UK) for $60 \mathrm{~min}$. Immunofluorescence in the arteries was observed under a confocal laser-scanning microscope (CLSM510, Carl Zeiss GmbH) with an excitation filter system $\left(458488^{-1} \mathrm{~nm}\right.$ for fluorescein-5-isothiocyanate) and an emission filter system $(543 \mathrm{~nm}$ for Cy3). Two fluorescence views were obtained from the same microscopic field.

\section{Immunohistochemical analysis}

The immunostaining density of nNOS-like immunoreactive (nNOS-LI) nerve fibers was analyzed using the method described by Hobara et al..$^{20,21}$ As the fluorescence intensity differed depending on the day of the experiment, the mesenteric arteries from 2K1C-RHRs and sham-operated rats were isolated, fixed and immunostained at the same time on the same day and mounted on the same slide glass. For quantitative evaluation of nNOS-LI, confocal projection images of nNOS immunostaining were magnified $\times 20$, digitized as TIF images using a digital camera system (Olympus SP-1000, Olympus, Tokyo, Japan) and imported into a Windows XP computer (Toshiba, Tokyo, Japan). The stored digital images were analyzed using image-processing software (Simple PCI; Compix, Imaging Systems, Cranberry Township, PA, USA). A measured field of $100 \mu \mathrm{m} \times 100 \mu \mathrm{m}\left(10000 \mu \mathrm{m}^{2}\right)$, which contained the adventitia layer including immunostained perivascular nerve fibers, was randomly selected on magnified images of the whole mount artery. The objective areas command was used to calculate the percentage of nNOS-LI-positive areas. The average density in three arteries was taken as the nerve density per animal.

To determine the number of nNOS-LI fibers, five horizontal lines were drawn on the image of a blood vessel in the same region where the density was estimated by computer analysis. Then, the number of fibers that crossed each line was counted, and the average of the number in three arteries was taken as the total number of fibers per animal.

\section{Statistics}

All values are expressed as the mean \pm s.e.m. Statistical analysis was performed using unpaired Student's $t$-test between two groups and one-way analysis of variance followed by Tukey's test for multiple groups. A value of $P<0.05$ was considered statistically significant.

\section{RESULTS}

Changes in systolic blood pressure and heart rate in 2K1C-RHRs The systolic blood pressure in 2K1C-RHRs, 4 weeks after the right renal artery occlusion, was significantly increased compared with that of sham-operated rats $(179.5 \pm 3.3 \mathrm{~mm} \mathrm{Hg}$ in $2 \mathrm{~K} 1 \mathrm{C}-\mathrm{RHRs}, n=22$; $98.3 \pm 3.6 \mathrm{~mm} \mathrm{Hg}$ in sham-operated rats, $n=21, P<0.01)$. There was no significant change in heart rate between 2K1C-RHRs (369.4 \pm 9.1 beats $\left.\mathrm{min}^{-1}\right)$ and sham-operated rats $\left(354.9 \pm 7.4\right.$ beats $\left.\mathrm{min}^{-1}\right)$.

\section{Vasoconstrictor responses to PNS and noradrenaline injection in 2K1C-RHRs and sham-operated rats}

As shown in Figure 1, PNS (2, 4 and $8 \mathrm{~Hz})$ of rat-perfused mesenteric vascular beds, without an endothelium and with resting tone frequency dependent, increased the perfusion pressure because of vasoconstriction in $2 \mathrm{~K} 1 \mathrm{C}-\mathrm{RHRs}$ and sham-operated rats $(2 \mathrm{~Hz}, 5.2 \pm 0.5$ and $3.3 \pm 0.3 \mathrm{~mm} \mathrm{Hg} ; 4 \mathrm{~Hz}, 11.9 \pm 1.4$ and $6.1 \pm 0.6 \mathrm{~mm} \mathrm{Hg} ; 8 \mathrm{~Hz}$, $54.6 \pm 6.3$ and $25.4 \pm 4.1 \mathrm{~mm} \mathrm{Hg}$, respectively, $n=10$ in 2K1C-RHRs and sham-operated rats). Vasoconstrictor responses to PNS at $2 \mathrm{~Hz}$ $(P<0.01), 4 \mathrm{~Hz}(P<0.01)$ and $8 \mathrm{~Hz}(P<0.01)$ were significantly higher in 2K1C-RHRs compared with sham-operated rats (Figure 1c). Bolus injections of noradrenaline $(0.5$ and $1.0 \mathrm{nmol})$ into the perfusate also caused concentration-dependent vasoconstriction in both $2 \mathrm{~K} 1 \mathrm{C}-\mathrm{RHRs}(n=10)$ and sham-operated rats $(n=10$; $0.5 \mathrm{nmol}, 28.2 \pm 5.6$ and $10.3 \pm 1.9 \mathrm{~mm} \mathrm{Hg} ; 1.0 \mathrm{nmol}, 59.3 \pm 9.8$ and $28.4 \pm 3.3 \mathrm{~mm} \mathrm{Hg}$, respectively). Vasoconstrictor responses to norepinephrine injection at concentrations of $0.5 \mathrm{nmol}(P<0.01)$ and $1.0 \mathrm{nmol}(P<0.01)$ were also significantly higher in $2 \mathrm{~K} 1 \mathrm{C}-\mathrm{RHRs}$ compared with sham-operated rats (Figure 1d).

Repeated PNS and noradrenaline injections caused reproducible vasoconstrictor responses. In the control response, the ratios of $S_{1}$ and $S_{2}$ at 2,4 and $8 \mathrm{~Hz}$ and $I_{1}$ and $I_{2}$ at 0.5 and $1.0 \mathrm{nmol}$ were $0.90 \pm 0.12$, $1.14 \pm 0.10,1.19 \pm 0.09$ and $1.01 \pm 0.10,1.08 \pm 0.05$ in $2 \mathrm{~K} 1 \mathrm{C}$-RHRs $(n=10)$ and $1.11 \pm 0.11,1.08 \pm 0.18,1.14 \pm 0.04$ and $1.01 \pm 0.15$, $1.10 \pm 0.13$ in sham-operated rats $(n=10)$, respectively. There was no significant difference in the ratio of PNS and noradrenaline responses between $2 \mathrm{~K} 1 \mathrm{C}-\mathrm{RHR}$ and sham-operated rats.

At the end of the experiment, successful removal of the endothelium was confirmed by the lack of a relaxant effect after a bolus injection of $1 \mathrm{nmol}$ acetylcholine in preparations with active tone produced by perfusion of Krebs solution containing methoxamine (Figure 2).

\section{Effects of L-NAME on vasoconstrictor responses to PNS and noradrenaline injection}

As shown in Figures 2 and 3, in perfused mesenteric vascular beds without an endothelium, the perfusion of L-NAME $(100 \mu \mathrm{M})$ did not alter the resting tone. In the presence of L-NAME, vasoconstrictor responses to PNS at 2, 4 and $8 \mathrm{~Hz}$ were significantly augmented in both $2 \mathrm{~K} 1 \mathrm{C}-\mathrm{RHRs}$ and sham-operated rats (ratio of 2, 4, $8 \mathrm{~Hz}$; $1.33 \pm 0.13, \quad 1.38 \pm 0.10, \quad 1.35 \pm 0.13$ in 2K1C-RHRs $(n=13)$; $2.01 \pm 0.19,2.12 \pm 0.21,1.97 \pm 0.14$ in sham-operated rats $(n=12)$ ). As shown in Figure $3 \mathrm{a}$, the augmenting effect of L-NAME on vasoconstriction in response to PNS in 2K1C-RHRs was significantly smaller than that in sham-operated rats. However, L-NAME did not affect the vasoconstriction induced by exogenously applied noradrenaline in either 2K1C-RHRs or sham-operated rats (Figure $3 \mathrm{~b}$ ). 
a
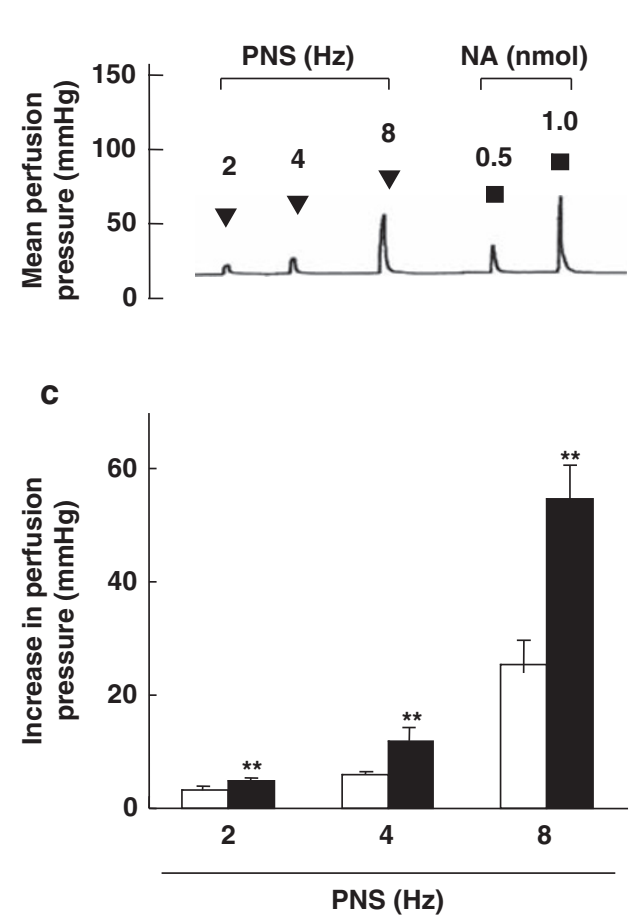

b

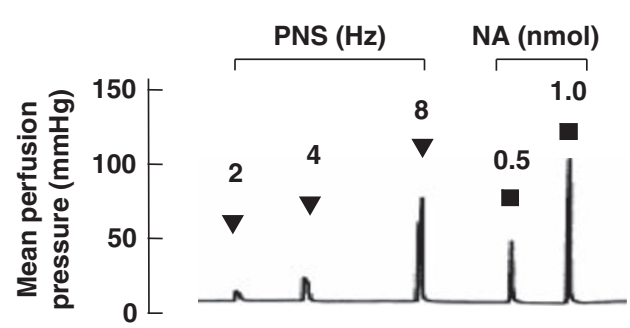

d

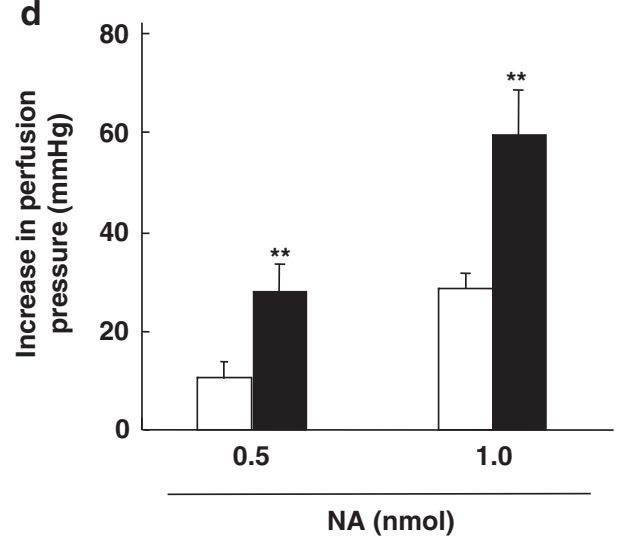

Sham $(n=10)$

2K1C-RHR (n=10)

Figure 1 Typical records (a, b) and bar graphs (c, d) showing vasoconstrictor responses to periarterial nerve stimulation (PNS; $\mathbf{\nabla})$ and bolus injection of noradrenaline (NA; $\mathbf{\square})$ in the perfused mesenteric vascular beds of sham-operated rats (a) and 2K1C-RHRs (b). (c, d) Indicate PNS- and NA injectioninduced responses, respectively. Each bar represents mean \pm s.e.m. (2K1C-RHRs, $n=10$; sham-operated rats, $n=10$ ). ${ }^{* *} P<0.01$ compared with shamoperated rats.

\section{Noradrenaline release in the perfusate}

As shown in Figure $4 \mathrm{a}$, in perfused mesenteric vascular beds without an endothelium and with resting tone, noradrenaline was detected in the perfusate. The basal amounts of noradrenaline in 2K1C-RHR and sham-operated rat preparations were $385.3 \pm$ $134.2 \mathrm{pg} \mathrm{ml}^{-1}(n=8)$ and $436.6 \pm 48.0 \mathrm{pg} \mathrm{ml}^{-1}(n=6)$, respectively. There was no difference in the basal release of noradrenaline between 2K1C-RHRs and sham-operated rats. The application of PNS at $8 \mathrm{~Hz}$ induced a significant increase in the amount of noradrenaline in the perfusate (net noradrenaline release: 2K1C-RHRs, $96.8 \pm 29.6 \mathrm{pg} \mathrm{ml}^{-1}(n=8)$; sham-operated rats, $42.0 \pm$ $\left.6.6 \mathrm{pg} \mathrm{ml}^{-1}(n=6)\right)$. A significant difference was found in PNSevoked noradrenaline release between 2K1C-RHRs and sham-operated rats. The perfusion of L-NAME $(100 \mu \mathrm{M})$ significantly enhanced the net PNS-evoked noradrenaline release in both $2 \mathrm{~K} 1 \mathrm{C}-\mathrm{RHR}$ and sham-operated rat preparations (2K1C-RHRs, $132.7 \pm 41.8 \mathrm{pg} \mathrm{ml}^{-1}$ $(n=8)$; sham-operated rats, $\left.77.2 \pm 9.2 \mathrm{pg} \mathrm{ml}^{-1}(n=6)\right)$. The augmenting effect of L-NAME on the PNS-evoked noradrenaline release in $2 \mathrm{~K} 1 \mathrm{C}-\mathrm{RHRs}$ was significantly smaller than that in sham-operated rats in Figure $4 \mathrm{~b}$.

\section{nNOS expression in the mesenteric artery}

As shown in Figure 5a, western blot analysis detected nNOS protein at $160 \mathrm{kDa}$ in the mesenteric arteries from 2K1C-RHRs and sham-operated rats. The protein expression of nNOS in $2 \mathrm{~K} 1 \mathrm{C}$ RHRs was significantly smaller than that in sham-operated rats (Figure 5b).

\section{Immunohistochemical study}

Immunohistochemical staining of the mesenteric artery showed dense innervation of adrenergic nNOS-LI fibers (Figure 6a) and TH-LI fibers (Figure 6b) in the network outside the mesenteric arteries. The innervation of TH-LI fibers was greater than that of nNOS-LI fibers (Figures $6 \mathrm{a}$ and $\mathrm{b}$ ). As shown in Figures $6 \mathrm{c}$ and d, double immunostainings showed that most nNOS-LI fibers in contact with the adrenergic TH-LI fibers appeared yellow when merged, indicating that there were close contact sites between the adrenergic and nitrergic nerves.

As shown in Figures $7 \mathrm{a}$ and $\mathrm{b}$, the immunohistochemical study showed dense innervation of nNOS-LI-containing fibers in the preparations of $2 \mathrm{~K} 1 \mathrm{C}-\mathrm{RHR}$ and sham-operated rats, and the density (Figure 7c) and number (Figure 7c) of nNOS-LI nerve fibers in 2K1CRHRs was $\sim 50 \%$ lower than that in sham-operated rats.

\section{DISCUSSION}

In this study, vasoconstrictions induced by PNS were augmented in the mesenteric arteries of 2K1C-RHRs compared with normotensive sham-operated rats. It has been reported that vasoconstriction induced by PNS of rat mesenteric vascular beds is abolished by tetrodotoxin (neurotoxin), guanethidine (adrenergic neuron blocker), prazosin $\left(\alpha_{1}\right.$-adrenoceptor antagonist) and 6-hydroxydopamine (adrenergic neuron destroyer). ${ }^{22,23}$ Therefore, it is very likely that the noradrenaline released from periarterial sympathetic adrenergic nerves mediates the PNS-induced vasoconstriction by activating postsynaptic $\alpha$-adrenoceptors. It has been reported that vasoconstrictor 


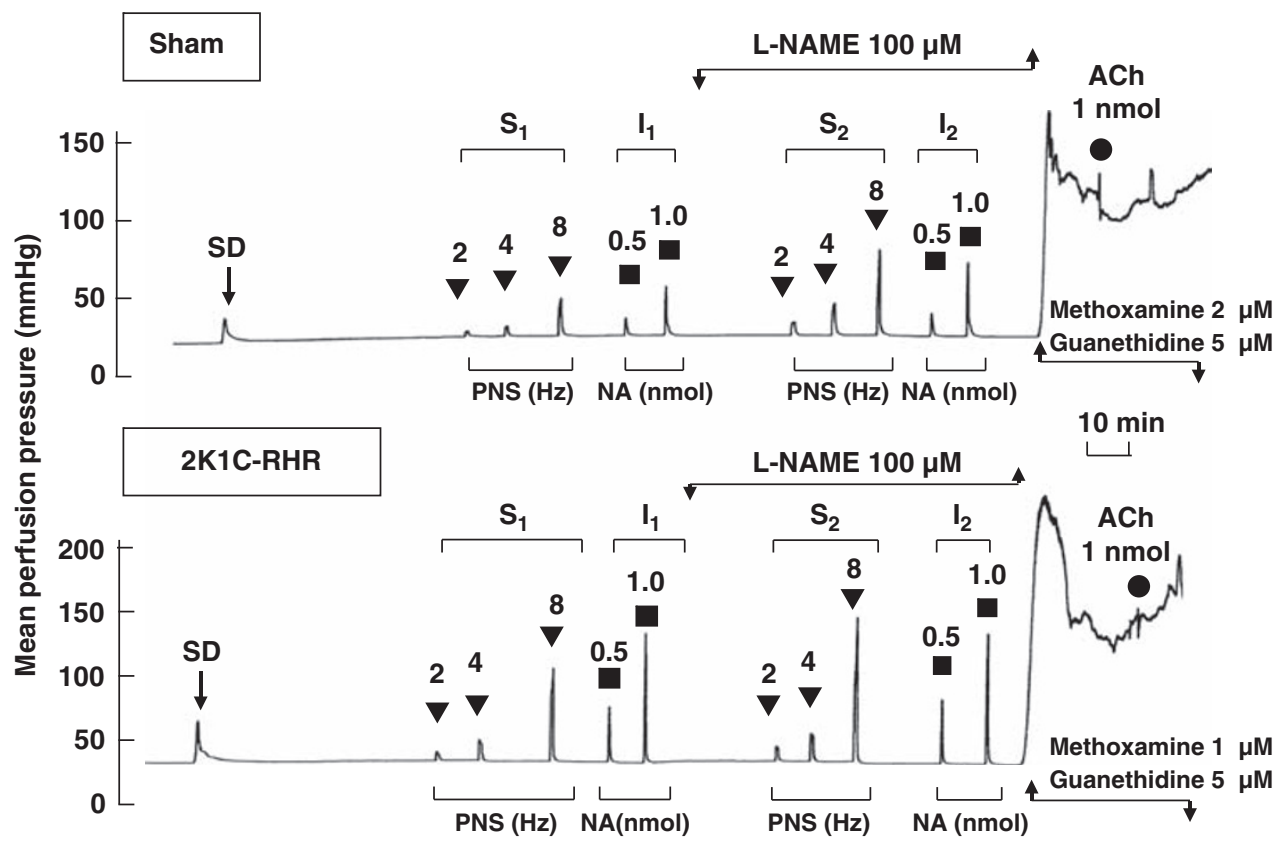

Figure 2 Typical records showing changes in vasoconstrictor responses to periarterial nerve stimulation (PNS; $\mathbf{\nabla}$ ) and bolus injection of noradrenaline (NA; 口) in the absence and presence of a nonselective NOS inhibitor, L-NAME, in the perfused mesenteric vascular beds of 2K1C-RHRs and sham-operated rats. $S_{1}$ and $I_{1}$ as well as $S_{2}$ and $I_{2}$ indicate responses to the first PNS and NA injection (control responses in the absence of L-NAME) as well as the second PNS and NA injection (responses in the presence of L-NAME), respectively. ACh; bolus injection of acetylcholine $(\bullet)$. L-NAME, N- $\omega$-nitro-L-arginine methyl ester; $\mathrm{SD}$, perfusion of sodium deoxycholate.
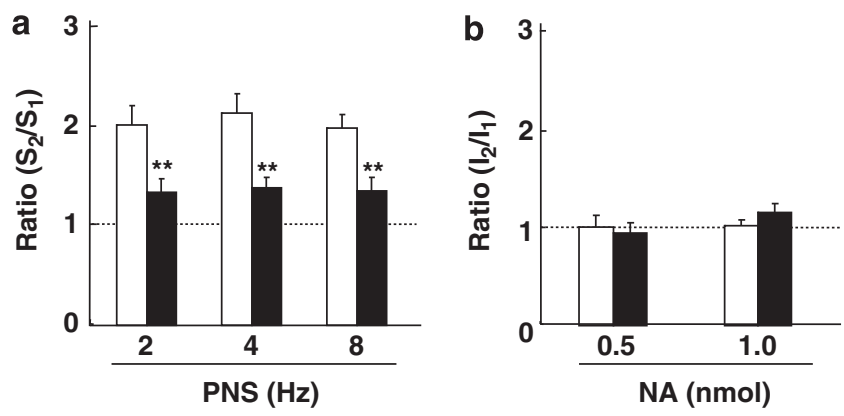

$\square$ Sham $(n=12) \quad$ 2K1C-RHR $(n=13)$

Figure 3 The effect of L-NAME on vasoconstrictor responses to periarterial nerve stimulation (PNS) (a) and bolus injection of noradrenaline (NA) (b) in the perfused mesenteric vascular beds of $2 \mathrm{~K} 1 \mathrm{C}-\mathrm{RHRs}$ and sham-operated rats. Ordinates show the ratio of $\mathrm{S}_{1}$ - and $\mathrm{S}_{2}$-induced vasoconstriction induced by PNS as well as the ratio of $I_{1}$ - and $I_{2}$-induced vasoconstriction induced by injection of NA. Values represent mean \pm s.e.m. ${ }^{* *} P<0.01$ compared with sham-operated rats.

responses to nerve stimulation and noradrenaline are significantly increased in the mesenteric arteries of $2 \mathrm{~K} 1 \mathrm{C}-\mathrm{RHRs} .{ }^{24}$ Vasoconstrictions induced by PNS have also been reported to be enhanced in the mesenteric vascular beds of SHRs. ${ }^{25}$ These findings support the present findings that the adrenergic nerve activity is enhanced in the mesenteric arteries of 2K1C-RHRs. In this study, the vasoconstriction induced by exogenously applied noradrenaline, which was mediated by postsynaptic $\alpha$-adrenoceptors, ${ }^{22}$ was significantly increased in the 2K1C-RHR preparations compared with those of sham-operated rats. Therefore, it is likely that increased $\alpha$-adrenoceptor activity also contributes to augmented PNS-induced vasoconstriction in 2K1C-RHRs.
The previous study showed that a nonselective NOS inhibitor, L-NAME, augmented the vasoconstrictor response to PNS in rats' denuded mesenteric arteries without affecting exogenously injected noradrenaline-induced vasoconstriction. ${ }^{6}$ These findings were confirmed by this study, in which vasoconstriction induced by PNS, but not noradrenaline injection, was enhanced by L-NAME in denuded mesenteric arteries of both 2K1C-RHRs and shamoperated rats. Additionally, L-NAME enhanced the PNS-induced noradrenaline release in the preparations taken from $2 \mathrm{~K} 1 \mathrm{C}-\mathrm{RHRs}$ and sham-operated rats. As the endothelium in these preparations had been removed, it appears that the augmentation by L-NAME results from the inhibition of nNOS. This suggests that neuronal NO, probably released from nitrergic nerves, presynaptically inhibits noradrenaline release from adrenergic nerve terminals in mesenteric arteries.

In this study, the augmenting effect of L-NAME on the vasoconstriction induced by PNS was decreased in 2K1C-RHR preparations compared with sham-operated rat preparations, suggesting that the inhibitory modulation of neuronal NO in adrenergic neurotransmission is decreased in $2 \mathrm{~K} 1 \mathrm{C}$-RHRs. It has been reported that $\mathrm{NO}$ acts as an inhibitory modulator for adrenergic neurotransmission under physiological conditions ${ }^{26}$ and that the NO-mediated modulation of adrenergic neurotransmission is blunted in the mesenteric arterial bed of SHRs. ${ }^{27}$ Furthermore, increased oxidative stress has been shown to occur in hypertension, ${ }^{28,29}$ and this increased oxidative stress causes a decrease in NO bioavailability. ${ }^{30}$ Taken together, these studies and the present findings suggest that the decrease in $\mathrm{NO}$ availability at adrenergic nerve synapses in hypertension results in facilitated adrenergic neurotransmission and the reduced effect of L-NAME on PNSinduced vasoconstriction. Thus, it seems likely that the altered modulation of adrenergic neurotransmission caused by neuronal $\mathrm{NO}$ contributes to the onset and maintenance of hypertension in 2K1C-RHRs. 


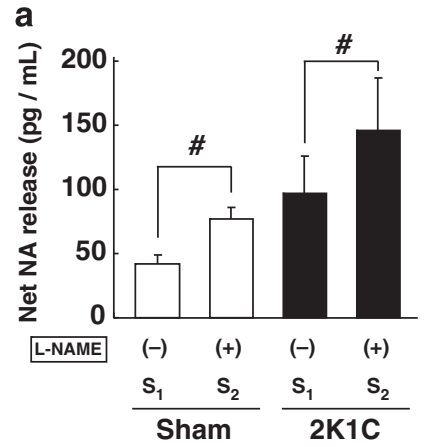

b

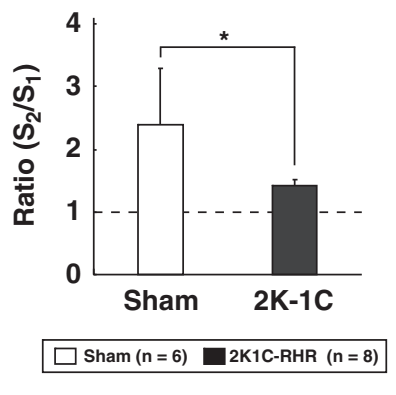

Figure 4 The effect of L-NAME on noradrenaline (NA) release evoked by periarterial nerve stimulation (PNS; $8 \mathrm{~Hz}$ ) in the perfused mesenteric vascular beds of $2 \mathrm{~K} 1 \mathrm{C}-\mathrm{RHRs}$ and sham-operated rats without an endothelium. In (a), the ordinates show the net release of NA (post PNS minus pre-PNS). In (b), the ordinates show the ratio of net NA release induced by PNS $\left(S_{1}\right)$ in the absence of L-NAME to the net NA release induced by PNS $\left(S_{2}\right)$ in the presence of L-NAME. Values represent mean \pm s.e.m. L-NAME, $N$ - $\omega$-nitro-L-arginine methyl ester. ${ }^{*} P<0.05$ compared with sham-operated rats. ${ }^{\#} P<0.05$, compared with $\mathrm{S}_{1}$.

a

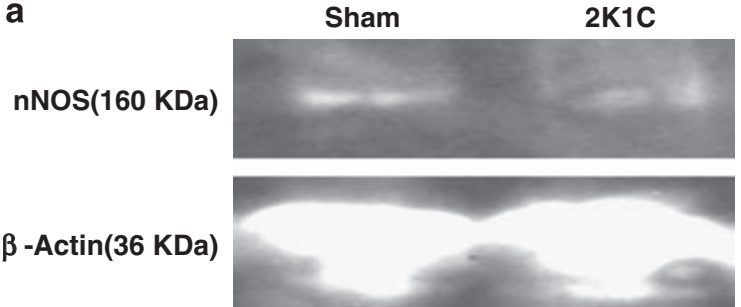

b

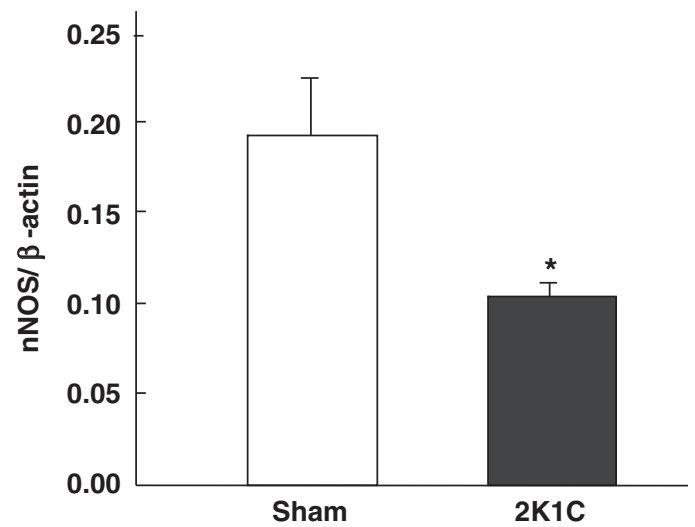

Figure 5 Western blot analysis for the expression of neuronal nitric oxide synthase (nNOS) protein in rat mesenteric arteries isolated from $2 \mathrm{~K} 1 \mathrm{C}$-RHRs and sham-operated rats. The nNOS protein was detected as $160 \mathrm{kDa}$ (a). In (b), each bar indicates mean \pm s.e.m. The ordinates indicate the fold increase over the $\beta$-actin values (sham-operated rats, $n=5 ; 2 \mathrm{~K} 1 \mathrm{C}-\mathrm{RHRs}$, $n=4) .{ }^{*} P<0.01$ compared with sham-operated rats.

In this study, we show that the expression of nNOS was markedly decreased in mesenteric arteries taken from 2K1C-RHRs compared with that taken from sham-operated rats. Furthermore, the immunohistochemical study showed that the density of nNOS-immunopositive nerve fibers was markedly reduced in the mesenteric arteries of 2K1C-RHRs, supporting the present findings that neuronal NO modulation in adrenergic neurotransmission is decreased in $2 \mathrm{~K} 1 \mathrm{C}$ RHRs. It has been reported that nNOS expression is decreased in the adrenal glands of $\mathrm{SHR}^{31}$ and that soluble guanylate cyclase, the key
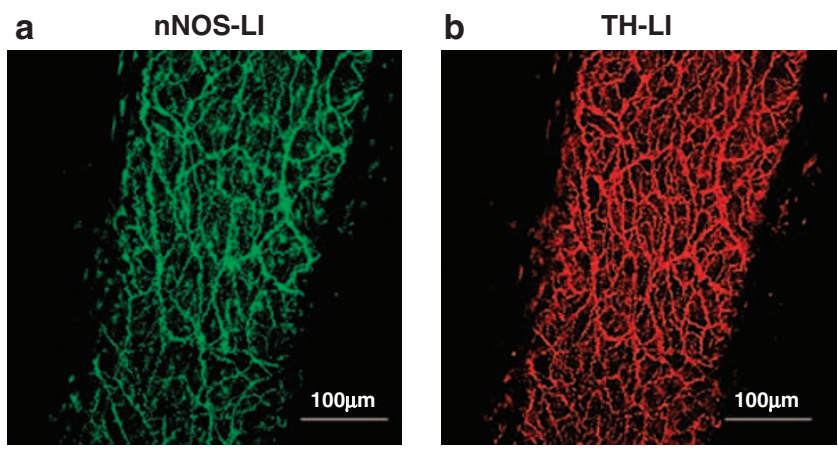

\section{c nNOS-LI+TH-LI}

d nNOS-LI+TH-LI

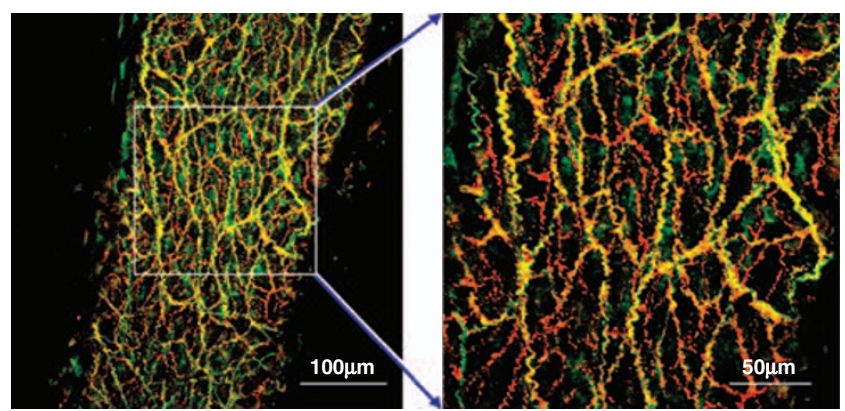

Figure 6 Confocal laser photomicrographic images showing the innervation of perivascular nerves in the mesenteric arteries of sham-operated rats. The upper images show the neuronal nitric oxide synthase ( $\mathrm{nNOS}$ ) (a, green)-like immunoreactivity (LI)- and tyrosine hydroxides (TH) (b, red)-LI-containing fibers. The lower images of (c) and (d) (which is a magnified image of the square area in c) are superimposed images of (a) and (b). The horizontal bars indicate $100 \mu \mathrm{m}(\mathbf{a}, \mathbf{b}, \mathbf{c})$ and $50 \mu \mathrm{m}(\mathbf{d})$. a Sham

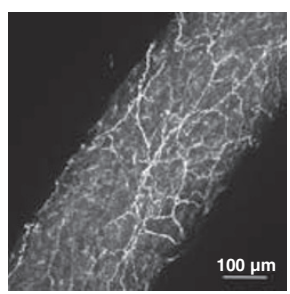

C

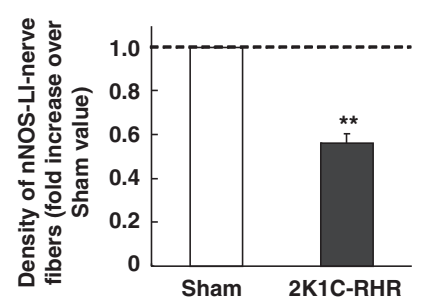

\section{b 2K1C-RHR}

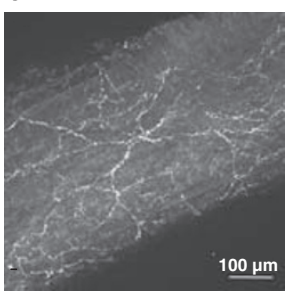

d

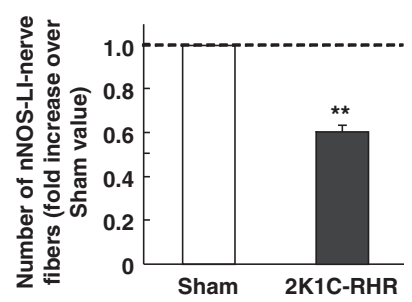

Figure 7 Confocal laser micrographs (a, b) and bar graphs (c, d) showing neuronal nitric oxide synthase (nNOS)-like immunoreactivity (LI)-containing fibers and changes in density (c) and number (d) of nNOS-LI innervation in sham-operated rats (a) and 2K1C-RHRs (b). The scale bar in each panel indicates $100 \mu \mathrm{m}$. In (c) and (d), the vertical scale indicates the fold increase over the value of sham-operated rats. Each bar indicates mean \pm s.e.m. (sham-operated rats, $n=4 ; 2$ K1C-RHRs, $n=4$ ). ${ }^{* *} P<0.01$ compared with sham-operated rats.

precursor of NO cyclic guanosine monophosphate-dependent effects, is downregulated in the aorta ${ }^{32}$ and atria ${ }^{33}$ of SHRs. The reninangiotensin system is strongly augmented in 2K1C-RHRs. The active 
substance of renin-angiotensin system, angiotensin II, is a potent vasoconstrictor, having strong effects on vascular tone regulation, facilitating sympathetic neurotransmission ${ }^{34}$ and reducing the bioavailability of $\mathrm{NO}$ as a result of increasing oxidative stress. $^{28,3,31}$ Furthermore, it has been reported that high blood pressure causes increased oxidative stress in the vasculature. ${ }^{35}$ Therefore, it is very likely that the acute increase in renin-angiotensin system and high blood pressure in 2K1C-RHRs blunts the inhibitory modulation of $\mathrm{NO}$-containing nerves in adrenergic neurotransmission and thereby leads to further facilitation of adrenergic neurotransmission.

Recently, artificial upregulation of nNOS through gene transfer in SHRs has been reported to result in the suppression of sympathetic hyperactivity by inhibiting noradrenaline release. ${ }^{36}$ This implies that the neuronal NO produced by $\mathrm{nNOS}$ has an important role in modulating the neuronal noradrenaline release from adrenergic nerves. In the present immunohistochemical study, double immunostainings showed both nNOS-immunopositive nerve fibers and THimmunopositive nerve fibers in the same neurons of the rat mesenteric artery. This finding strongly suggests that vascular adrenergic nerves have coalescence sites with nNOS-containing nitrergic nerves. It is likely that these coalescence sites are close contact areas between adrenergic and nitrergic nerves and that both nerves interact at these areas.

In conclusion, the present results suggest that the inhibitory function of nitrergic nerves on adrenergic neurotransmission is significantly decreased in 2K1C-RHRs. This functional alteration, based on a decrease in nNOS expression and nitrergic innervation, leads to enhanced adrenergic neurotransmission and contributes to the initiation and development of renovascular hypertension.

\section{CONFLICT OF INTEREST}

The authors declare no conflict of interest.

\section{ACKNOWLEDGEMENTS}

This work was supported in part by a Grant-in-Aid for Scientific Research (KAKENHI No. 21590095) from the Ministry of Education, Science and Technology of Japan.

1 Kawasaki H, Takasaki K, Saito A, Goto K. Calcitonin gene-related peptide acts as a novel vasodilator neurotransmitter in mesenteric resistance vessels of the rat. Nature 1988; 335: 164-167.

2 Toda N, Okamura T. Mechanism of neurally induced monkey mesenteric artery relaxation and contraction. Hypertension 1992; 19: 161-166.

3 Macarthur H, Westfall TC, Wilken GH. Oxidative stress attenuates NO-induced modulation of sympathetic neurotransmission in the mesenteric arterial bed of spontaneously hypertensive rats. Am J Physiol Heart Circ Physiol 2008; 294: H183-H189.

4 Shiraki H, Kawasaki H, Tezuka S, Nakatsuma A, Kurosaki Y. Endogenous calcitonin gene-related peptide (CGRP) mediates adrenergic-dependent vasodilation induced by nicotine in mesenteric resistance arteries of the rat. Br J Pharmacol 2000; 130 1083-1091.

5 Bredt DS, Hwang PM, Snyder SH. Localization of nitric oxide synthase indicating a neural role for nitric oxide. Nature 1990; 347: 768-770.

6 Hatanaka Y, Hobara N, Honghua J, Akiyama S, Nawa H, Kobayashi Y, Takayama F, Gomita $Y$, Kawasaki H. Neuronal nitric-oxide synthase inhibition facilitates adrenergic neurotransmission in rat mesenteric resistance arteries. J Pharmacol Exp Ther 2006; 316: 490-497.

7 Toda N, Okamura T. Nitroxidergic nerve: regulation of vascular tone and blood flow in the brain. J Hypertens 1996; 14: 423-434.

8 Breslow MJ, Tobin JR, Bredt DS, Ferris CD, Snyder SH, Traystman RJ. Role of nitric oxide in adrenal medullary vasodilation during catecholamine secretion. Eur J Pharmacol 1992; 210: 105-106.

9 Yamamoto R, Wada A, Asada Y, Yuhi T, Yanagita T, Niina H, Sumiyoshi A. Functional relation between nitric oxide and noradrenaline for the modulation of vascular tone in rat mesenteric vasculature. Naunyn Schmiedebergs Arch Pharmacol 1994; 349: 362-366.
10 Vo PA, Reid JJ, Rand MJ. Endothelial nitric oxide attenuates vasoconstrictor responses to nerve stimulation and noradrenaline in the rat tail artery. Eur J Pharmacol 1991; 199: 123-125.

11 Woodman OL, Pannangpetch P. Enhancement of noradrenergic constriction of large coronary arteries by inhibition of nitric oxide synthesis in anaesthetized dogs. $\mathrm{Br} \mathrm{J}$ Pharmacol 1994; 112: 443-448.

12 Anderson EA, Sinkey CA, Lawton WJ, Mark AL. Elevated sympathetic nerve activity in borderline hypertensive humans. Evidence from direct intraneural recordings. Hypertension 1989; 14: 177-183.

13 Esler M, Ferrier C, Lambert G, Eisenhofer G, Cox H, Jennings G. Biochemical evidence of sympathetic hyperactivity in human hypertension. Hypertension 1991; 17: III29-III35.

14 Westfall TC, Meldrum MJ. Alterations in the release of norepinephrine at the vascular neuroeffector junction in hypertension. Annu Rev Pharmacol Toxicol 1985; 25 : 621-641.

15 Katholi RE, Whitlow PL, Winternitz SR, Oparil S. Importance of the renal nerves in established two-kidney, one clip Goldblatt hypertension. Hypertension 1982; 4: 166-174.

16 Fujita M, Ando K, Nagae A, Fujita T. Sympathoexcitation by oxidative stress in the brain mediates arterial pressure elevation in salt-sensitive hypertension. Hypertension 2007; 50: 360-367.

17 Tsuda K, Kuchii M, Nishio I, Masuyama Y. Neurotransmitter release, vascular responsiveness and their suppression by $\mathrm{Ca}$-antagonist in perfused mesenteric vasculature of DOCA-salt hypertensive rats. Clin Exp Hypertens A 1986; 8: 259-275.

18 Kawasaki H, Nuki C, Saito A, Takasaki K. Role of calcitonin gene-related peptidecontaining nerves in the vascular adrenergic neurotransmission. J Pharmacol Exp Ther 1990; 252: 403-409.

19 Takenaga M, Kawasaki H. Endogenous calcitonin gene-related peptide suppresses vasoconstriction mediated by adrenergic nerves in rat mesenteric resistance blood vessels. Eur J Pharmacol 1999; 367: 239-245.

20 Hobara N, Gessei-Tsutsumi N, Goda M, Takayama F, Akiyama S, Kurosaki Y, Kawasaki $\mathrm{H}$. Long-term inhibition of angiotensin prevents reduction of periarterial innervation of calcitonin gene-related peptide (CGRP)-containing nerves in spontaneously hypertensive rats. Hypertens Res 2005; 28: 465-474.

21 Hobara N, Goda M, Kitamura Y, Takayama F, Kawasaki H. Innervation and functional changes in mesenteric perivascular calcitonin gene-related peptide- and neuropeptide Y-containing nerves following topical phenol treatment. Neuroscience 2006; 141: 1087-1099.

22 Kawasaki H, Takasaki K. Vasoconstrictor response induced by 5-hydroxytryptamine released from vascular adrenergic nerves by periarterial nerve stimulation. $J$ Pharmacol Exp Ther 1984; 229: 816-822.

23 Kawasaki H, Urabe M, Takasaki K. Enhanced 5-hydroxytryptamine release from vascular adrenergic nerves in spontaneously hypertensive rats. Hypertension 1987; 10: 321-327.

24 Eikenburg DC, Lokhandwala MF. Effects of captopril on vascular noradrenergic transmission during the development of two-kidney, one clip Goldblatt hypertension in rats. J Hypertens 1983; 1: 115-122.

25 Draper AJ, Meghji S, Redfern PH. Enhanced presynaptic facilitation of vascular adrenergic neurotransmission in spontaneously hypertensive rats. J Auton Pharmacol 1989; 9: 103-111.

26 Kolo LL, Westfall TC, Macarthur H. Nitric oxide decreases the biological activity of norepinephrine resulting in altered vascular tone in the rat mesenteric arterial bed. $\mathrm{Am}$ J Physiol Heart Circ Physiol 2004; 286: H296-H303.

27 Kolo LL, Westfall TC, Macarthur H. Modulation of neurotransmitter release by NO is altered in mesenteric arterial bed of spontaneously hypertensive rats. Am J Physiol Heart Circ Physiol 2004; 287: H1842-H1847.

28 Griendling KK, Minieri CA, Ollerenshaw JD, Alexander RW. Angiotensin II stimulates NADH and NADPH oxidase activity in cultured vascular smooth muscle cells. Circ Res 1994; 74: 1141-1148.

29 Zalba G, San Jose G, Moreno MU, Fortuno MA, Fortuno A, Beaumont FJ, Diez J. Oxidative stress in arterial hypertension: role of NAD(P)H oxidase. Hypertension 2001; 38: 1395-1399.

30 Nava E, Farre AL, Moreno C, Casado S, Moreau P, Cosentino F, Luscher TF. Alterations to the nitric oxide pathway in the spontaneously hypertensive rat. J Hypertens 1998; 16: 609-615.

31 Qadri F, Arens T, Schwartz EC, Hauser W, Dominiak P. Angiotensin-converting enzyme inhibitors and AT1-receptor antagonist restore nitric oxide synthase (NOS) activity and neuronal NOS expression in the adrenal glands of spontaneously hypertensive rats. Jpn J Pharmacol 2001; 85: 365-369.

32 Ruetten H, Zabel U, Linz W, Schmidt HH. Downregulation of soluble guanyly cyclase in young and aging spontaneously hypertensive rats. Circ Res 1999; 85: 534-541.

33 Heaton DA, Lei M, Li D, Golding S, Dawson TA, Mohan RM, Paterson DJ. Remodeling of the cardiac pacemaker L-type calcium current and its beta-adrenergic responsiveness in hypertension after neuronal NO synthase gene transfer. Hypertension 2006; 48: 443-452.

34 Kawasaki H, Cline Jr WH, Su C. Enhanced angiotensin-mediated facilitation of adrenergic neurotransmission in spontaneously hypertensive rats. J Pharmacol Exp Ther 1982; 221: 112-116.

35 Dworakowski R, Alom-Ruiz SP, Shah AM. NADPH oxidase-derived reactive oxygen species in the regulation of endothelial phenotype. Pharmacol Rep 2008; 60: 21-28.

36 Li D, Wang L, Lee CW, Dawson TA, Paterson DJ. Noradrenergic cell specific gene transfer with neuronal nitric oxide synthase reduces cardiac sympathetic neurotransmission in hypertensive rats. Hypertension 2007; 50: 69-74. 